\title{
Persistencia de efectos a largo plazo de la fertilización fosfatada y la introducción de leguminosas en pastizales del Uruguay
}

del Pino, A., Lezama, F., Pezzani, F. y Parodi, G.

DOI: $10.31047 / 1668.298 x . v 38 . n 1.26856$

\begin{abstract}
RESUMEN
En el campo natural (CN) de Uruguay la introducción de leguminosas con fertilización fosfatada $(\mathrm{CN}+\mathrm{LP})$ evita restricciones nutricionales, pero son poco conocidos sus efectos sobre el suelo y la pastura. Para evaluar estos efectos en el largo plazo se utilizó un experimento previo de introducción de Lotus corniculatus y fertilización fosfatada con un tratamiento testigo de CN (1995-2002). Entre 2013 y 2015 se determinó la producción primaria neta aérea (PPNA), contenido de nutrientes de la planta, composición y diversidad florística. En el suelo se analizó $\mathrm{pH}$, contenido de C orgánico, P disponible y orgánico. En dos gramíneas nativas se evaluó la colonización micorrícica. El suelo $\mathrm{CN}+\mathrm{LP}$ presentó mayor contenido de $\mathrm{P}$ disponible, pero no hubo diferencias en C orgánico. Aunque la PPNA fue similar, el contenido de P del forraje fue mayor en $\mathrm{CN}+\mathrm{LP}$ respecto a $\mathrm{CN}$, mientras que la micorrización fue mayor en CN. La composición florística y la diversidad no difirieron. Se concluye que, aunque no se mantuvieron las ventajas productivas de CN+LP en PPNA, hubo una mejora en la calidad del forraje debido a su mayor contenido de P. Sin embargo, no hubo efectos en servicios ecosistémicos claves como la diversidad de especies.
\end{abstract}

Palabras clave: nutrientes en planta; micorrizas; siembra en cobertura; producción primaria neta aérea (PPNA); diversidad.

del Pino, A., Lezama, F., Pezzani, F. y Parodi, G. 2021. Persistence of long-term effects of phosphate fertilization and legume introduction in grasslands of Uruguay. Agriscientia 38: 99-109

\section{SUMMARY}

In natural grasslands (NG) of Uruguay the introduction of legumes and $P$ fertilization $(N G+L P)$ prevents nutritional restrictions, but the long-term effects on soil and pasture are little known. To evaluate its long-term effects, a previous experiment was used, which involved the introduction of Lotus corniculatus and phosphate fertilisation with a control treatment of NG (1995-2002). Between 
2013 and 2015 the aboveground net primary production (ANPP), plant nutrient content, floristic composition and diversity were determined. In the soil, $\mathrm{pH}$, organic C content, available $\mathrm{P}$ and organic $\mathrm{P}$ were analyzed. The mycorrhizal colonization of two native grasses was evaluated. The NG+LP soil presented higher content of available $\mathrm{P}$, but there were not differences in organic C. Although ANPP was similar, the forage P content was higher in of Lotus corniculatus compared to NG, while the mycorrhizal colonization was higher in NG. Floristic composition and diversity did not differ. It is concluded that, although in the long term the ANPP increase was not sustained in the NG+LP treatment, there was an improvement in quality of the forage, due to its higher $P$ content. However, there was not any effect on provision of key ecosystem services, such as species diversity.

Keywords: nutrients in plant; mycorrhiza; reseeding; aboveground net primary production (ANPP); diversity.

del Pino, A. (ORCID: 0000-0002-2168-2579), Lezama, F. (ORCID: 0000-0002-1557-8011), Pezzani, F. (ORCID: 0000-0003-2156-1176) y Parodi, G. (ORCID: 0000-0003-4534-8900): Universidad de la República, Facultad de Agronomía. Garzón 780, Montevideo, Uruguay. Correspondencia a: amabelia@fagro.edu.uy

\section{INTRODUCCIÓN}

Recientemente Uruguay ha atravesado un cambio profundo en el uso del suelo, caracterizado principalmente por un marcado aumento de la superficie forestada y agrícola a expensas de la superficie cubierta por comunidades de pastizal o campo natural (CN). Estos cambios tienen impactos ambientales sobre la provisión de bienes y servicios ecosistémicos que afectan la calidad de vida de la sociedad y la forma en que ésta percibe y valora los cambios en el uso de la tierra (Paruelo et al., 2006). Además de constituir el soporte de la ganadería extensiva en el Uruguay, el campo natural proporciona otra serie de servicios ecosistémicos: secuestro de carbono, control climático, regulación de inundaciones, beneficios culturales, entre otros (Altesor, 2011).

En general, el CN está caracterizado por una predominancia de pastos estivales, una marcada estacionalidad de la producción y una limitada calidad forrajera (Millot, Risso y Methol, 1987). Las especies vegetales que lo componen están generalmente adaptadas a la escasez de $\mathrm{P}$, pudiendo crecer con muy bajos niveles de este nutriente. En consecuencia en el forraje de $\mathrm{CN}$ se han registrado concentraciones de $\mathrm{N}$ y $\mathrm{P}$ por debajo de los niveles de suficiencia para los animales (Berretta, 1998; del Pino, Andion y Rodriguez Olivera, 2015). La introducción de leguminosas con fertilización fosfatada sobre campo natural (CN+LP) (localmente denominados mejoramientos extensivos o siembra en cobertura) supera las restricciones nutricionales más importantes (disponibilidad de N y P) y representa una tecnología de bajo costo y fácil adopción (Risso, 1991). Para estos fines, varias especies del género Lotus han demostrado una buena adaptación a distintos tipos de suelos (Ayala y Carámbula, 2009). Se ha postulado además que el aumento en producción de biomasa de los CNLP conlleva un mayor aporte de $\mathrm{C}$ al suelo, ya sea a través del mayor crecimiento de raíces como del aporte de restos secos (Conant, Paustian y Elliott, 2001).

$\mathrm{Si}$ bien generalmente se acepta que la introducción de leguminosas y fertilización fosfatada es más compatible con la conservación de la biodiversidad del CN que su reemplazo por agricultura $\mathrm{O}$ pasturas sembradas, no existe consenso en cuanto a su efecto sobre la composición de especies a largo plazo (Risso, 1991). Frecuentemente los CN+LP muestran corta persistencia, con pérdida de las leguminosas sembradas y aumento de malezas, especialmente Cynodon dactylon (Millot et al., 1987; Jaurena et al., 2016). La baja persistencia puede deberse a los efectos diferenciales de los nutrientes adicionados sobre las especies vegetales, resultando generalmente en una promoción de especies anuales de rápido crecimiento (Jaurena et al. 2016). Las leguminosas aportan $\mathrm{N}$ a la pastura a través 
de la fijación biológica, pero se ha observado que el enriquecimiento con $\mathrm{N}$ afecta negativamente en forma directa la diversidad vegetal de los pastizales naturales (Tilman, 1993). Además, asociado a este incremento, aumenta frecuentemente la invasión de especies exóticas, lo cual a su vez representa una amenaza para la conservación de la biodiversidad local por el desplazamiento de especies nativas por parte de las invasoras (Gurevitch y Padilla, 2004; Melbourne et al., 2007). Los efectos del $P$ son menos claros en este sentido, ya que se han reportado datos contradictorios en cuanto a su efecto sobre la conservación de especies de los pastizales (Ceulemans, Merckx, Hens y Honnay, 2013). Lo que sí es claro, es que la fertilización fosfatada afecta negativamente las interacciones micorrícicas, particularmente la colonización de las raíces de las plantas por hongos micorrizógenos arbusculares (HMA) (Kahiluoto, Ketoja, Vestberg y Saarela, 2001; Boaming y Beaver, 2016). En Uruguay si bien son escasos los estudios sobre HMA en CN, se han observado estas tendencias (García, Pezzani, Rodríguez y del Pino, 2016).

Dado que la investigación sobre los CN+LP ha abarcado básicamente la instalación y los primeros años de producción, este trabajo se plantea como objetivo responder a dos interrogantes: ¿se mantienen en el largo plazo las ventajas agronómicas que justificaron su implementación?, ¿cuáles son los efectos a largo plazo sobre algunos de los servicios ecosistémicos que provee el CN? Para responderlos se estudió el efecto residual de un experimento con CN+LP luego de 18 años de su instalación sobre la producción y contenido de nutrientes de la biomasa aérea y la diversidad de especies. Dado que la fertilización con $\mathrm{P}$ es un aspecto clave de los CN+LP, para evaluar su residualidad se analizaron variables relacionadas a la dinámica del P (P disponible, P orgánico, índice de retención de $\mathrm{P}$ y grado de micorrización de dos gramíneas), así como cambios en la materia orgánica del suelo (MO).

\section{MATERIALES Y MÉTODOS}

\section{Sitio experimental}

El estudio se realizó en el Departamento de Salto, Uruguay ( $31^{\circ} 14^{\prime} \mathrm{S}, 57^{\circ} 08^{\prime} \mathrm{O}$ ), en un sitio donde se llevó a cabo entre 1995 y 2002 un experimento que tuvo como objetivo la cuantificación del efecto de la fertilización fosfatada y la introducción de leguminosas sobre la calidad de las pasturas y su relación con la producción de carne de los animales en pastoreo (del Pino et al., 2015). Este experimento se instaló sobre un suelo profundo desarrollado sobre materiales derivados de basalto, siendo los parámetros del horizonte $\mathrm{A}$ : densidad aparente: $1,1 \mathrm{~g} \mathrm{~cm}^{-3} ; \mathrm{pH}: 5,7$; C orgánico: $34,8 \mathrm{~g} \mathrm{~kg}^{-1} ; \mathrm{N}$ orgánico: 0,36 \%; P Bray N. ${ }^{\circ} 1: 4$ mg kg $^{-1}$; óxidos de Fe: 1,8\%. En 1995 se delimitaron 15 parcelas, de 2 ha cada una, en las que se dispusieron en cinco bloques al azar los tres tratamientos: 1) testigo de campo natural (CN); 2) siembra en cobertura de Lotus corniculatus, con agregado de $26 \mathrm{~kg} \mathrm{ha}^{-1}$ de $\mathrm{P}$ a la instalación y $13 \mathrm{~kg} \mathrm{ha}^{-1}$ de P como dosis de refertilización en el otoño de los años siguientes (nivel medio de P: P1); 3) siembra en cobertura con dosis de $39 \mathrm{~kg} \mathrm{ha}^{-1}$ y $26 \mathrm{~kg} \mathrm{ha}^{-1}$ de $\mathrm{P}$ de instalación y refertilización respectivamente (nivel alto de P: P2). A través de estas fertilizaciones anuales entre 1996 y 1999, recibieron en total dosis de 72 y $144 \mathrm{~kg} \mathrm{ha}^{-1}$ de $\mathrm{P}$ en los tratamientos P1 y P2 respectivamente. Se utilizaron fuentes de P solubles, aplicándose superfosfato de calcio común a la siembra y en las fertilizaciones de 1996 y 1997, en tanto que las restantes fertilizaciones se utilizó superfosfato de calcio triple. En 1997 en CN+LP se sembró trébol blanco (Trifolium repens), el cual perduró por dos años, a diferencia del $L$. corniculatus, el cual se mantuvo productivo hasta 2001. Entre julio de 1996 y febrero de 2002 se registró la información detallada de producción de forraje, así como de las ganancias de peso vivo de los terneros en pastoreo. En el 2002 se retiraron las cercas de las parcelas, las cuales pasaron a formar parte del manejo general del establecimiento y el sitio ha sido pastoreado conjuntamente por ovinos y vacunos hasta el 2013. Para el presente estudio se utilizaron nueve parcelas del experimento previo correspondientes a tres de los cinco bloques.

\section{Muestreos y análisis químicos}

Muestreo y análisis de suelos. En octubre de 2013 en cada parcela se tomaron muestras compuestas de 15 tomas en cada profundidad (05, 5-10 y 10-15 cm). El P disponible se determinó por el método Bray 1 (Bray y Kurtz, 1945). En las muestras superficiales $(0-5 \mathrm{~cm})$ se determinaron el contenido de $\mathrm{P}$ orgánico (PO) mediante la técnica descrita por O'Halloran (1993) según la cual el contenido de $\mathrm{P}$ orgánico se calcula como la diferencia entre el contenido de $\mathrm{P}$ extraído con $\mathrm{H}_{2} \mathrm{SO}_{4} 0,5 \mathrm{M}$ de muestras calcinadas a $550{ }^{\circ} \mathrm{C}$ y sin calcinar. El índice de retención de $\mathrm{P}$ (IRP) se determinó de acuerdo a Sims (2009). Brevemente se agitó el suelo por $16 \mathrm{~h}$ a $25{ }^{\circ} \mathrm{C}$ con una solución de $\mathrm{K}_{2} \mathrm{HPO}_{4}$ con $100 \mathrm{mg} \mathrm{L}^{-1}$ de $\mathrm{P}$ y $\mathrm{CaCl}_{2}$ 0,01 M (relación suelo:solución 1:10, con una concentración de $\mathrm{P}$ equivalente a $1000 \mathrm{mg} \mathrm{kg}^{-1}$ de 
suelo). Se determinó el P remanente en solución, calculándose por diferencia el $\mathrm{P}$ retenido por el suelo.

En la capa de suelo de 0 a $15 \mathrm{~cm}$ se determinaron: $\mathrm{pH}$, contenido de carbono orgánico (CO) por oxidación con dicromato de potasio (Nelson y Sommers,1996). Los cationes intercambiables se analizaron mediante extracción con acetato de amonio neutro y posterior determinación por emisión (K) y absorción atómica (Ca y Mg), según Isaac y Kerber (1971).

Estimación de la productividad primaria neta aérea y análisis de tejido vegetal. Para determinar la productividad primaria neta aérea (PPNA) se utilizaron cuatro jaulas de exclusión de pastoreo de $1,0 \times 0,5 \mathrm{~m}$ por parcela, las cuales se rotaron dentro de la parcela durante la evaluación. La estimación de producción de forraje se realizó mediante cortes a $2 \mathrm{~cm}$ de altura (método de doble muestreo) (Lynch, 1951) en forma estacional desde abril de 2014 hasta marzo de 2015. Los cortes se realizaron el 5/4/2014, 1/7/2014, 1/10/2014 y 20/12/2014 y 20/03/2015. El forraje cosechado se secó y se molió. A partir del peso seco de forraje cosechado y del área de las jaulas se calculó el rendimiento en materia seca (MS) expresándose en kg ha-1. Para su análisis se calcinaron las muestras en mufla a $550^{\circ} \mathrm{C}$ por $5 \mathrm{~h}$, se disolvieron las cenizas en ácido clorhídrico $(\mathrm{HCl})$ y en esos extractos se realizó la determinación colorimétrica de $\mathrm{P}$ (Murphy y Riley, 1962) y determinación de cationes (K, Ca y Mg) como fue descrito. El contenido de $\mathrm{N}$ se analizó según el método de Kjeldahl (Nelson y Sommers, 1973).

Muestreo florístico. En diciembre de 2013 se evaluó la composición de especies mediante subparcelas de 1,0 x 1,0 m distribuidas en forma sistemática (seis subparcelas en cada parcela). En cada subparcela se registraron todas las especies presentes, y a cada una se le asignó un valor de cobertura-abundancia siguiendo la escala de Braun-Blanquet (Mueller-Dombois y Ellenberg, 1974). El muestreo se realizó a fines de primavera porque la mayor parte de las especies presentan estructuras reproductivas que permiten su identificación a campo.

Muestreo de raíces para análisis de micorrizas. Se seleccionaron gramíneas nativas de buena calidad forrajera, que estuvieran presentes en todas las parcelas y que han presentado diferente respuesta a la disponibilidad de P. En estudios previos (García et al., 2016; Jaurena et al., 2016) Coelorhachis selloana redujo su frecuencia en parcelas fertilizadas, y por lo tanto es considerada una especie decreciente al $\mathrm{P}$, mientras que Paspalum dilatatum no varió su frecuencia (especie indiferente al P). Se realizaron dos muestreos, en diciembre de 2013 (verano) y en junio de 2014 (invierno), tomando muestras de raíces de cinco individuos de cada especie por parcela. Para observar la colonización por HMA las raíces fueron teñidas según la técnica de Koske y Gemma (1989) y se observaron en un microscopio óptico para cuantificar las estructuras fúngicas. Se estimó el porcentaje de colonización micorrícica total como el número de segmentos colonizados respecto al total, multiplicado por 100. Asimismo, se cuantificó por separado la colonización de las estructuras fúngicas: vesículas (estructuras de almacenamiento de los hongos micorrizógenos arbusculares), arbúsculos y ovillos.

\section{CÁLCULOSY ANÁLISIS ESTADÍSTICOS}

Los resultados de los parámetros de suelo ( $\mathrm{P}$ disponible, $\mathrm{CO}, \mathrm{PO}, \mathrm{IRP}$ ) fueron analizados mediante análisis de varianza de bloques al azar, con separación de medias por el método de Tukey para determinar las diferencias entre los tratamientos (CN, P1 y P2). En cuanto a la PPNA y la concentración de nutrientes en el forraje en cada corte se calculó el promedio de las cuatro jaulas para cada parcela. A partir del resultado de cada parcela se realizó el análisis estadístico mediante el procedimiento Proc Mixed con mediciones repetidas (muestreos estacionales) para determinar el efecto de los tratamientos sobre la PPNA, así como los contenidos foliares de P, N, relación N/P y contenido de cationes ( $\mathrm{K}$, Ca y Mg).

A partir de los datos florísticos se calcularon la riqueza de especies $(S=$ número de especies por parcela) y el índice de diversidad de ShannonWeiner:

$$
H^{\prime}=\sum \text { pi Inpi }
$$

donde pi corresponde a la frecuencia relativa de la especie i). Los efectos de los tratamientos sobre los índices de diversidad se analizaron mediante análisis de varianza de bloques al azar. Las diferencias en composición de especies entre tratamientos fueron evaluadas mediante Multiresponse Permutation Procedure (MRPP) (Biondini, Mielke y Berry, 1988). El MRPP provee un estadístico delta, que representa el promedio ponderado de las distancias intra grupo y está asociado a un p-valor que indica la probabilidad de observar un delta igual o menor que el observado al azar (McCune y Mefford, 1999).

La presencia de micorrizas en cada especie se analizó para las variables micorrización total, 
vesículas y ovillos, mediante análisis de varianza con los factores estación del año (verano e invierno) y tratamiento de fertilización. Los registros de arbúsculos fueron muy escasos en todos los tratamientos por lo cual no se pudo hacer el análisis estadístico. Todos los análisis mencionados se realizaron con el programa SAS 9.2 (2009).

\section{RESULTADOS Y DISCUSIÓN}

\section{Fósforo en el suelo}

El $\mathrm{P}$ disponible en el suelo mostró una clara estratificación (Figura 1), siendo en la capa 0-5 cm mayor en $\mathrm{CN}+\mathrm{LP}$ respecto al $\mathrm{CN}(\mathrm{p}<0,05)$, aunque no hubo diferencias entre P1 y P2. No se registró efecto de los tratamientos en las otras profundidades de muestreo. Estos resultados evidencian que la aplicación en superficie del fertilizante fosfatado mostró una escasa residualidad, ya que aún en la capa superficial del suelo los niveles son relativamente bajos, en comparación con niveles críticos para la producción de leguminosas (Bordoli, 1998). Asimismo, en este tipo de suelo es esperable un limitado incremento del $\mathrm{P}$ disponible como consecuencia de la fertilización, debido a su gran capacidad de retención de P. Hernández, Otegui y Zamalvide (1995) en su caracterización de formas de $P$ en suelos del Uruguay atribuyeron este comportamiento al alto contenido de óxidos de Fe de los suelos desarrollados sobre materiales derivados de basalto.

En contraste con el $\mathrm{P}$ disponible, el índice

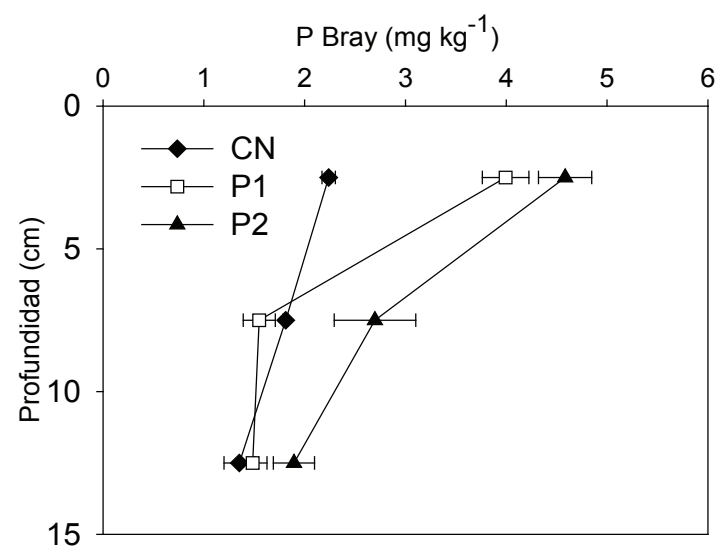

Figura 1. Distribución de $\mathrm{P}$ disponible del suelo (Bray $\mathrm{N} .^{\circ}$ 1) en profundidad ( $\mathrm{mg} \mathrm{kg}^{-1} \pm$ error estándar). Efecto residual de la introducción de leguminosas y fertilización fosfatada.CN: campo natural; P1: fertilización fosfatada media; P2: fertilización fosfatada alta de retención de $\mathrm{P}$ (IRP) no fue afectado por la fertilización. Los valores de IRP (promedio 632 mg $\mathrm{kg}^{-1}$ ) fueron sensiblemente mayores a los citados por Gutiérrez Boem, Alvarez, Cabello y Fernández (2008) para suelos de la región pampeana de Argentina (promedio $329 \mathrm{mg} \mathrm{kg}^{-1}$ ). La fertilización fosfatada tiende a disminuir la capacidad de retención de $\mathrm{P}$ por saturación de los sitios de retención, como fue observado por Rubio, Cabello, Gutiérrez Boem y Munaro (2008) en suelos de Argentina, pero esto no ocurrió en nuestro estudio, probablemente debido a la relativamente baja cantidad de $\mathrm{P}$ agregado.

Los tratamientos de fertilización no mostraron efectos sobre el contenido de PO, aunque se constató que la fertilización promovió el enriquecimiento en $\mathrm{P}$ de la $\mathrm{MO}$, el cual está inversamente relacionado al cociente CO:PO. El contenido de PO del suelo, independientemente del tratamiento, fue mayor al reportado por Hernández, Otegui y Zamalvide (1995) para CN de vertisoles desarrollados sobre basalto (271 y $277 \mathrm{mg} \mathrm{kg}^{-1}$ ). No obstante, la mayor profundidad de muestreo utilizada por estos autores $(0-15 \mathrm{~cm})$ relativiza la comparación, ya que probablemente el PO tenga un patrón de estratificación, disminuyendo en profundidad. También Pascale, Heredia y Giufré (2000) reportan niveles menores de $\mathrm{PO}(0-7 \mathrm{~cm})$ en un relevamiento de vertisoles de Entre Ríos, Argentina (promedio para CN de $204 \mathrm{mg} \mathrm{kg}^{-1}$ ). En cambio, el enriquecimiento en $\mathrm{P}$ de la materia orgánica del suelo (relación $\mathrm{CO}: \mathrm{PO}$ ) se encuentra dentro del rango citado por Hernández et al. (1995) para 31 suelos del Uruguay (promedio de 169,7 $\mathrm{mg} \mathrm{kg}^{-1}$ ). En nuestro estudio la relación significativamente menor en P2 respecto a los otros tratamientos sugiere que la fertilización realizó una contribución al PO del suelo, aunque la misma no se reflejó en aumentos del nivel de $\mathrm{PO}$, sino al enriquecimiento en $\mathrm{P}$ de la $\mathrm{MO}$. El significado del $\mathrm{PO}$ en términos de nutrición de las plantas no es claro, pero dado su abundancia es esperable que la mineralización de una pequeña parte del PO resulte en un aporte importante de P, contribuyendo a la absorción de $\mathrm{P}$ por la pastura en CN+LP. Es así que Morón (1996) reporta en pasturas fertilizadas aumentos en la concentración de $\mathrm{P}$ de la $\mathrm{MO}$, especialmente en la biomasa microbiana, sugiriendo que PO sufre procesos de mineralización y acumulación similares a los del C y el $\mathrm{N}$ orgánico, pudiendo ser una fuente de $\mathrm{P}$ para la pastura.

Al analizar el proceso en su conjunto puede plantearse que, debido al alto IRP de los suelos, el $\mathrm{P}$ agregado como fertilizante fue inicialmente retenido mediante adsorción y precipitación por 
compuestos de Ca y Fe. No obstante, con el tiempo esta adición de $\mathrm{P}$ resultó en un enriquecimiento de las formas de $\mathrm{P}$ disponibles y del contenido de $\mathrm{P}$ de la materia orgánica, aunque este incremento fue escaso debido a la discontinuación de la fertilización.

\section{Carbono orgánico en el suelo}

El incremento en producción vegetal de $\mathrm{CN}+\mathrm{LP}$ no tuvo un efecto residual de largo plazo sobre el contenido de $\mathrm{CO}$ en la capa de 0 a $15 \mathrm{~cm}$ del suelo. Estos resultados coinciden con los de Bondaruk, Lezama, del Pino y Piñeiro (2020) quienes detectaron escasas diferencias en el contenido de $\mathrm{C}$ del suelo en 12 sitios CN+LP de Lotus subbiflorus en comparación con el $\mathrm{CN}$ adyacente. El contenido de CO del suelo mostró un marcado decrecimiento en profundidad alcanzando en la capa de $0-5 \mathrm{~cm}$ valores de aproximadamente el doble del promedio de las otras dos capas (Figura 2). En términos generales el resultado de $C N+L P$ sobre el $\mathrm{CO}$ del suelo no es claro. Por un lado se espera que el aumento en PPNA conlleve un ingreso de $\mathrm{C}$ al sistema, con incorporación de $\mathrm{C}$ proveniente de raíces y hojarasca al humus del suelo (Dubeux, Sollenberger, Mathews, Scholeberg y Santos, 2007). Por otro lado, la entrada de N y P al sistema podría producir un enriquecimiento en nutrientes del humus, haciéndolo más susceptible a la descomposición, con pérdida neta de C (Lodge, King y Harden, 2006). También se ha postulado que cada suelo tiene una limitada capacidad de acumulación de C, por lo cual en el

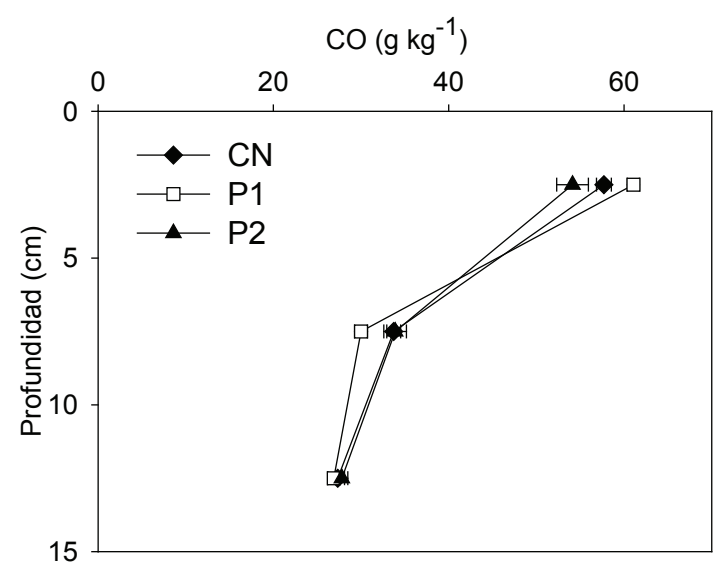

Figura 2. Distribución del carbono orgánico (CO) del suelo en profundidad $\left(\mathrm{g} \mathrm{kg}^{-1} \pm\right.$ error estándar). Efecto residual de la introducción de leguminosas y fertilización fosfatada. CN: campo natural; P1: fertilización fosfatada media; P2: fertilización fosfatada alta
CN no es de esperar un aumento, por tratarse de una situación cercana al equilibrio. En contraste, en suelos que han perdido C por laboreos o larga historia de agricultura, el establecimiento de pasturas permitiría aumentar el CO hasta llegar a ese nivel de equilibrio (Stewart, Paustian, Conant, Plante y Six, 2007). El hecho de que no se haya encontrado efecto de CN+LP sobre el contenido total de $\mathrm{C}$ del suelo, no significa que se haya mantenido constante la constitución de la MO. Los trabajos de Piñeiro, Paruelo, Jobbágy, Jackson y Oesterheld (2009) y Bondaruk et al. (2020) reportan cambios en las fracciones de la $\mathrm{MO}$, aunque no se modifiquen los contenidos totales, observando que el efecto neto de ganancia o pérdida de $\mathrm{C}$ de las pasturas mejoradas depende del tipo de suelo y del manejo.

No se observaron efectos de los tratamientos sobre otras propiedades del suelo examinadas $(\mathrm{pH}$, contenido de cationes intercambiables) en ninguna de las profundidades de muestreo (datos no presentados). Estas propiedades tampoco mostraron tendencias claras en estratificación y sus niveles fueron similares a los medidos en el muestreo original del suelo del experimento en 1995.

\section{Productividad primaria neta aérea y contenido de nutrientes en el forraje}

La producción de biomasa aérea no presentó diferencias significativas entre tratamientos en ninguna de las estaciones (Figura 3). La suma de las PPNA estacionales que representa la producción anual, tampoco presentó diferencias entre tratamientos (4429; 4629 y $4446 \mathrm{~kg} \mathrm{ha}^{-1}$ de

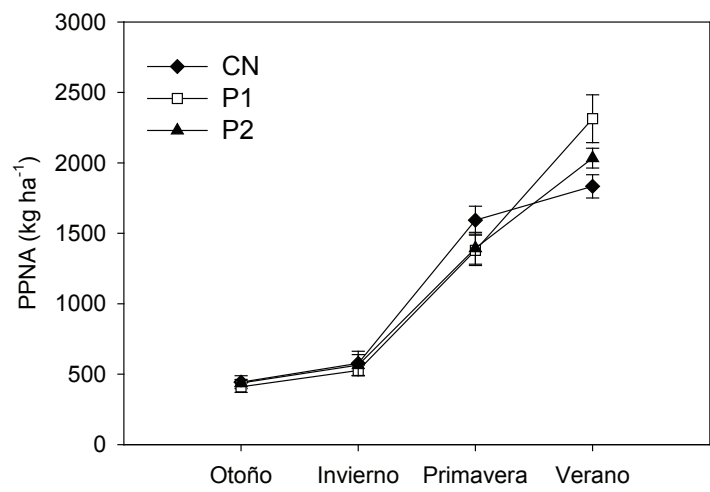

Figura 3. Efecto residual de la introducción de leguminosas y fertilización fosfatada sobre la producción estacional de biomasa aérea ( $\mathrm{kg} \mathrm{ha}^{-1} \pm$ error estándar). CN: campo natural; P1: fertilización fosfatada media; P2: fertilización fosfatada alta 
Tabla 1. Índice de retención de $\mathrm{P}$ (IRP), contenido de P orgánico (PO) y relación $\mathrm{CO}: \mathrm{PO}$ en la capa de 0-5 cm del suelo. Efecto residual de la introducción de leguminosas y fertilización fosfatada. CN: campo natural; P1: fertilización fosfatada media; P2: fertilización fosfatada alta. Letras distintas en la misma columna indican diferencias significativas $(p<0,05)$

\begin{tabular}{llll}
\hline Tratamiento & IRP & PO & CO:PO \\
\hline \multicolumn{4}{c}{ mg kg $^{-1}$} \\
CN & $630 \mathrm{a}$ & $309 \mathrm{a}$ & $187 \mathrm{a}$ \\
P1 & $623 \mathrm{a}$ & $328 \mathrm{a}$ & $186 \mathrm{a}$ \\
P2 & $644 \mathrm{a}$ & $325 \mathrm{a}$ & $166 \mathrm{~b}$ \\
\hline
\end{tabular}

MS para CN, P1 y P2 respectivamente). En todos los tratamientos hubo una clara estacionalidad en la producción de biomasa aérea, con una muy baja producción otoño-invernal y una alta producción estival. Si bien en este último período la producción del $\mathrm{CN}$ resultó menor a la de $\mathrm{CN}+\mathrm{LP}$, las diferencias no alcanzaron significación estadística.

La concentración de $\mathrm{P}$ en la biomasa aérea mostró el efecto positivo de los tratamientos de fertilización, con diferencias $(p<0,05)$ entre $C N$ y $C N+L P$, así como entre dosis de $P$ dentro de los tratamientos de CN+LP. No se encontraron diferencias en la concentración de $\mathrm{N}$ en la biomasa aérea (Figura 4). No hubo diferencias entre tratamientos en la concentración de cationes (Mg, $\mathrm{K}$ y $\mathrm{Ca}$ ) en el forraje cosechado (datos no presentados). La relación N:P de la biomasa aérea cosechada mostró valores decrecientes en los tratamientos $(14,3 ; 12,6$ y 11,1 para $C N$, $\mathrm{P} 1$ y $\mathrm{P} 2$ respectivamente), aunque se observaron diferencias $(p<0,05)$ solamente entre la relación $\mathrm{N}: \mathrm{P}$ del $\mathrm{CN}$ y $\mathrm{CN}+\mathrm{LP}$, pero no entre dosis de $P(p=0,10)$. Estos valores sugieren una mayor limitación de la PPNA por la disponibilidad de N en relación al P (Koerselman y Meuleman, 1996).

La acumulación de nutrientes en el forraje cosechado (suma de los cuatro cortes) solamente mostró diferencias estadísticas para $\mathrm{P}$, aunque también las cantidades de $\mathrm{N}, \mathrm{Mg}$ y K absorbidas por las plantas durante el año fueron mayores en $\mathrm{CN}+\mathrm{LP}$ a las del CN (Tabla 2).

Tabla 2. Contenido de $\mathrm{P}, \mathrm{N}, \mathrm{K}, \mathrm{Ca}$ y $\mathrm{Mg}$ en biomasa aérea de pasturas (total anual acumulado). Efecto residual de la introducción de leguminosas y fertilización fosfatada. CN: campo natural; P1: fertilización fosfatada media; P2: fertilización fosfatada alta. Letras distintas en la misma columna indican diferencias significativas $(p<0,05)$

\begin{tabular}{lccccc}
\hline Tratamiento & $\mathrm{P}$ & $\mathrm{N}$ & $\mathrm{K}$ & $\mathrm{Ca}$ & $\mathrm{Mg}$ \\
\hline \multicolumn{5}{c}{$\mathrm{kg} \mathrm{ha}^{-1}$} \\
CN & $3,9 \mathrm{a}$ & $53,0 \mathrm{a}$ & $30,1 \mathrm{a}$ & $25,2 \mathrm{a}$ & $7,7 \mathrm{a}$ \\
P1 & $5,3 \mathrm{ab}$ & $59,6 \mathrm{a}$ & $33,8 \mathrm{a}$ & $27,3 \mathrm{a}$ & $8,2 \mathrm{a}$ \\
P2 & $5,7 \mathrm{~b}$ & $62,9 \mathrm{a}$ & $32,4 \mathrm{a}$ & $24,6 \mathrm{a}$ & $8,5 \mathrm{a}$ \\
\hline
\end{tabular}

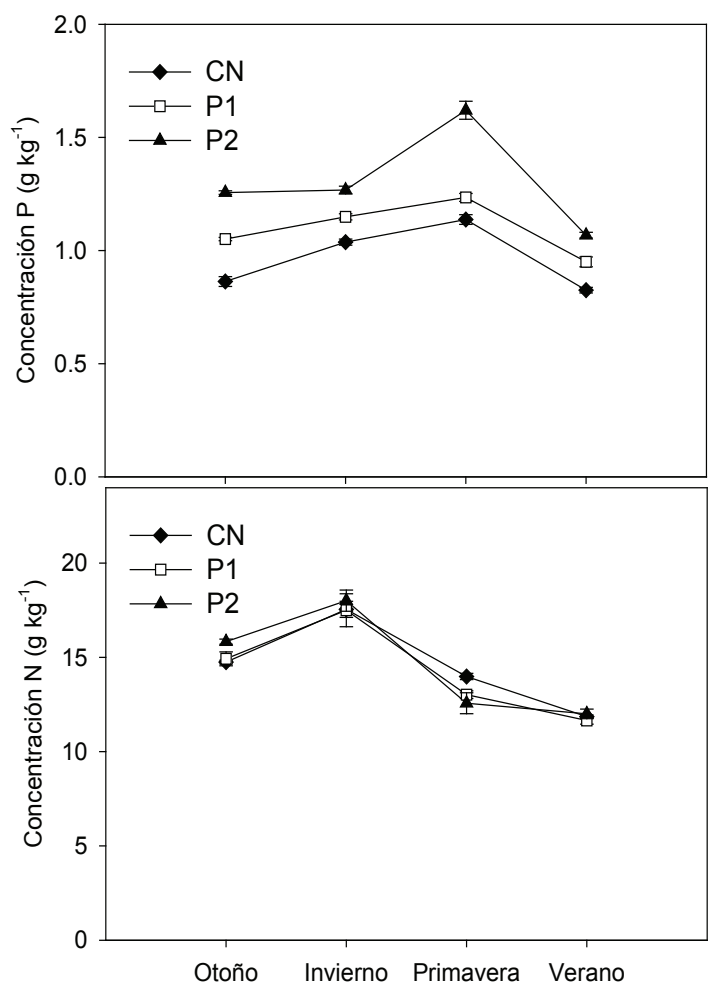

Figura 4. Efecto residual de la introducción de leguminosas y fertilización fosfatada sobre la concentración de P y N en la biomasa aérea ( $\mathrm{mg} \mathrm{g}^{-1} \pm$ error estándar). CN: campo natural; P1: fertilización fosfatada media; P2: fertilización fosfatada alta

Los cambios en la productividad forrajera que habían sido observados durante los primeros 7 años del experimento (del Pino et al., 2015) no fueron detectados 18 años después. En particular se perdió el aporte que representaban las leguminosas sembradas, las cuales llegaron a significar el $60 \%$ de la biomasa total producida. En cambio, las diferencias entre tratamientos en la concentración de $\mathrm{P}$ en la biomasa aérea y en la cantidad total de $\mathrm{P}$ absorbido, indican que, a pesar de la alta capacidad de retención de $\mathrm{P}$ del suelo, una parte del P agregado estuvo disponible para las plantas luego de 14 años de discontinuar la fertilización. La concentración de P en el forraje de $\mathrm{CN}$ (promedio 0,9 $\mathrm{g} \mathrm{kg}^{-1}$ ), presentó valores similares a los obtenidos por del Pino et al. (2015), estando por debajo del nivel crítico de $1,0 \mathrm{~g} \mathrm{~kg}^{-1}$ para los requerimientos animales de $P$ en pasturas (Karn, 2001), no así los valores promedio de $\mathrm{CN}+\mathrm{LP}$ (1,1 y $1,3 \mathrm{~g} \mathrm{~kg}^{-1}$ para $\mathrm{P} 1$ y $\mathrm{P} 2$ respectivamente). Estos resultados sugieren que los mejoramientos, a pesar de no incrementar la producción de forraje, mantienen una residualidad en la calidad y consecuentemente en su aptitud para la nutrición 
de los animales en pastoreo. En contraste, los tratamientos no afectaron la concentración de $\mathrm{N}$ en la biomasa aérea, lo que sugiere que el aporte de $\mathrm{N}$ de las leguminosas fue de corta duración, y la escasez de $\mathrm{N}$ es probablemente una de las causas de la escasa diferencia en producción de forraje entre los tratamientos.

\section{Composición y diversidad florística}

Las especies más importantes en términos de cobertura registradas fueron dos gramíneas estivales; entre ambas acumularon un $48 \%$ en promedio: Paspalum notatum $(32,7)$ y Axonopus affinis $(15,1)$. Las siguen en orden de importancia: Piptochaetium montevidense $(11,1)$, Bothriochloa laguroides. (6,2) y Stipa nessiana $(5,0)$. Contrariamente a lo esperado según los antecedentes, la composición florística no difirió entre los tratamientos (MRPP: $\Delta=-0,043 ; p=0,74$ ). Además, no se observó un reemplazo de especies perennes nativas por especies ruderales como se ha observado previamente (Jaurena et al., 2016); de hecho, todas las especies más importantes en términos de cobertura son gramíneas perennes. Por otro lado, no se constató la presencia de Cynodon dactylon en los relevamientos, ni de ninguna otra especie catalogada como invasora.

En el mismo sentido, la riqueza de especies a nivel de parcela no fue afectada por los tratamientos: esta presentó en promedio 31,2 especies $\mathrm{m}^{-2}$ $(F=0,296 ; p=0,75)$. Tampoco se observaron efectos sobre la diversidad de especies $(F=0,035$; $p=0,966$, medida a través del índice de Shannon).

Estos resultados contrastan fuertemente con los reportados no solo por un antecedente directo (Jaurena et al., 2016), sino también a lo esperado de acuerdo al aumento observado en disponibilidad de $\mathrm{P}$ en el suelo (Pañella, Cardozo, Cuadro, Reyno y Lezama, 2020) y la elevada biomasa de especies sembradas alcanzada en el transcurso de los primeros años del experimento (del Pino et al., 2015). Una de las explicaciones posibles reside en las características del suelo en el sitio de estudio. Se ha demostrado que propiedades como la textura y el $\mathrm{pH}$ influyen en el grado en que las comunidades responden a cambios en la disponibilidad de recursos (Harpole et al., 2016). En este sentido, el sitio presenta diferencias sustanciales en términos de textura y material parental con el área donde se realizó el estudio reportado por Jaurena et al. (2016). Podría hipotetizarse que la mayor capacidad de retención de $\mathrm{P}$ de los suelos de origen basálticos como los de este estudio, al capturar el $\mathrm{P}$, amortigua los efectos de la fertilización (Hernández et al., 1995). Comprender en dónde radican las diferencias en la resiliencia de las diferentes comunidades de pastizal se destaca como un aspecto clave para enfocar futuras investigaciones.

\section{Micorrizas}

Los efectos residuales de la introducción de leguminosas con fertilización fosfatada se manifestaron a través de un efecto negativo sobre la micorrización tanto de $P$. dilatatum como de $C$. selloana. Este efecto llega a reducir a la mitad la presencia de micorrizas en plantas de parcelas fertilizadas con dosis alta en relación al CN. Ambas gramíneas presentaron los mayores niveles de colonización micorrícica y de estructuras fúngicas (vesículas, ovillos y arbúsculos) en plantas provenientes del CN colectadas en verano, observándose el mayor efecto negativo de la fertilización en esa estación (Figura 5). En
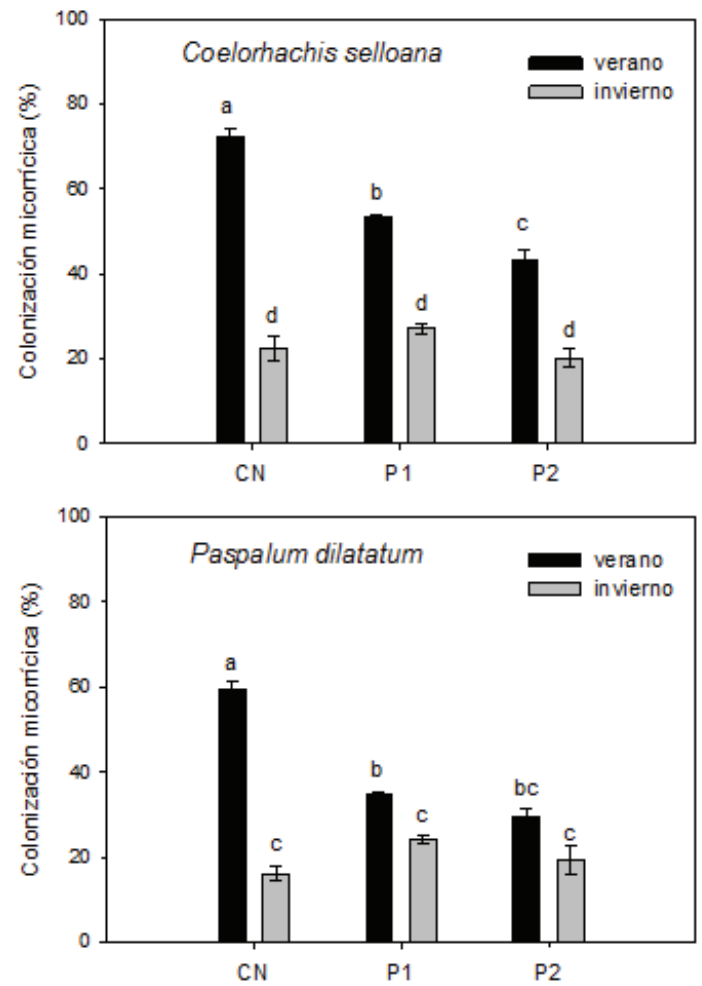

Figura 5. Efecto residual de la introducción de leguminosas y fertilización fosfatada sobre la colonización micorrícica total (\% \pm error estándar) en raíces de Coelorhachis selloana y Paspalum dilatatum en verano (barras negras) e invierno (barras grises). CN: campo natural; P1: fertilización fosfatada media; P2: fertilización fosfatada alta. Letras diferentes indican diferencias significativas $(p<0,05)$ entre estación y tratamientos para cada especie 
verano, en C. selloana el $\mathrm{P}$ redujo 30 y $40 \%$ la presencia de micorrizas en los tratamientos P1 y P2, respectivamente $(p<0,05)$. Asimismo hubo diferencias entre los dos niveles de fertilización $(p<0,05)$. En cambio en $P$. dilatatum la micorrización fue aproximadamente un $50 \%$ menor por efecto de la fertilización fosfatada ( $p<0,05$ en ambos casos), no registrándose diferencias entre los dos niveles de P. García et al. (2016) también encontraron que la presencia de micorrizas mostró un claro patrón estacional con mayores valores de colonización en los meses cálidos. Santos-González, Finlay y Tehler (2007) describieron la dinámica estacional de la interacción micorrícica, en parte relacionada con el estado fenológico y la actividad fotosintética de las plantas. En el caso de C. selloana y P. dilatatum se trata de gramíneas estivales lo que coincide con la mayor micorrización en verano así como también con la presencia de estructuras de intercambio de los HMA (arbúsculos y ovillos) en esa estación.

En cuanto a la presencia de vesículas en $C$. selloana fue menor también en verano por efecto de la fertilización fosfatada ( $p<0,05)$, pero en $P$. dilatatum no hubo efecto sobre esta variable. La presencia de estructuras de intercambio (ovillos y arbúsculos) fue muy escasa en ambas especies. En el caso de los ovillos los máximos registros se observaron en verano en plantas del $\mathrm{CN}$ siendo los registros 4,2 $\pm 0,4$ en $C$. selloana y 3,2 $\pm 0,6$ en $P$. dilatatum. En cambio, solamente se registraron escasos arbúsculos en verano en plantas de ambas gramíneas provenientes de las parcelas de $\mathrm{CN}$.

Los resultados de este trabajo coinciden con lo reportado por Kahiluoto et al. (2001) y Boaming y Beaver (2016) quienes sostienen que en ambientes con menor disponibilidad de $\mathrm{P}$ las interacciones micorrícicas se vieron favorecidas principalmente a través de una mayor asignación de recursos por parte de las plantas para recompensar a los hongos micorrizógenos, aumentando por esta vía la absorción de P. Al aumentar la disponibilidad de $\mathrm{P}$ podría existir un control fitocéntrico que restrinja la interacción teniendo en cuenta el costo energético que la misma representa para las plantas (Boaming y Beaver, 2016). García et al. (2016) también encontraron una disminución de la micorrización en $C$. selloana y $P$. dilatatum en respuesta al $\mathrm{P}$ en un experimento en la región este de Uruguay. En dicho experimento el P disponible en suelo luego de 15 años de fertilización fosfatada fue mayor que en el presente estudio ya que el $\mathrm{CN}$ contenía un $29 \%$ del P disponible de CN+LP (0-15 $\mathrm{cm}$ ), en tanto que en nuestro estudio el contenido de $\mathrm{P}$ disponible del suelo bajo $\mathrm{CN}$ representaba un $68 \%$ respecto a CN+LP. A pesar de este hecho la micorrización de ambas gramíneas se redujo menos que en este experimento en suelos desarrollados sobre basalto, y la máxima reducción fue en $P$. dilatatum de $25 \%$ y en $C$. selloana de 30 \% (García et al., 2016). Estas diferencias son probablemente debidas al tipo de suelo y la intensidad del pastoreo, que puede afectar a las micorrizas (Oehl et al., 2010).

\section{CONCLUSIONES}

Las ventajas agronómicas iniciales de la siembra de leguminosas con fertilización fosfatada se vieron atenuadas, en especial aquellas relacionadas al incremento de la PPNA y al aporte de $\mathrm{N}$ al sistema realizado por las leguminosas. No obstante, el incremento del contenido de $\mathrm{P}$ del forraje, indica que se trata de una pastura de mejor calidad, y en consecuencia con un mayor potencial productivo respecto al $\mathrm{CN}$.

En cuanto a los servicios ecosistémicos no se detectaron efectos a largo plazo del mejoramiento sobre la mayoría de las propiedades del suelo evaluadas, tampoco en la composición y diversidad de especies del pastizal. Dado que este resultado no es coincidente con los de estudios previos, queda planteado como interrogante cuál es el nivel de intensificación compatible con la conservación de la diversidad.

\section{AGRADECIMIENTOS}

Los autores agradecen a Fernando Gorriti, en cuyo establecimiento se realizó el experimento. También agradecen al Ing. Agr. Marcelo Pereira Machín por su colaboración en la planificación del estudio y en el muestreo florístico. Este proyecto fue financiado por Comisión Sectorial de Investigación Científica (CSIC) de la Universidad de la República Oriental del Uruguay.

\section{BIBLIOGRAFIA}

Altesor, A. (2011). Servicios ecosistémicos de los pastizales naturales. En A. Altesor, W. Ayala, J. M. Paruelo (Eds.), Bases ecológicas y tecnológicas para el manejo de pastizales (Serie Fondo de Promoción de Tecnología Agropecuaria 26, 221-234). Montevideo, Uruguay: Instituto Nacional de Investigación Agropecuaria (INIA).

Ayala, W. y Carámbula, M. (2009). El valor agronómico del género Lotus. Montevideo, Uruguay: Instituto Nacional de Investigación Agropecuaria (INIA). 
Berretta, E. J. (1998). Contenido de minerales en pasturas naturales de basalto. I. Especies nativas. En E. J. Berreta (Ed.), Seminario de Actualización en Tecnologías para Basalto (Serie Técnica 102, 63-73). Tacuarembó, Uruguay: Instituto Nacional de Investigación Agropecuaria (INIA).

Biondini, M. E., Mielke, P. W. y Berry, K. J. (1988). Datadependent permutation techniques for the analysis of ecological data. Vegetatio, 75 (3), 161-168.

Boaming, J. y Bever, J. (2016). Plant preferential allocation and fungal reward decline with soil phosphorus: implications for mycorrhizal mutualism. Ecosphere, 7 (5), 1-11. DOI: https://doi.org/10.1002/ecs2.1256

Bondaruk, V., Lezama, F., del Pino, A. y Piñeiro, G. (2020). Overseeding legumes in natural grasslands: Impacts on root biomass and soil organic matter of commercial farms. Science of the Total Environment, 743, 140771. DOI: https://doi.org/10.1016/j.scitotenv.2020.140771

Bordoli, J. M. (1998). Fertilización de pasturas de leguminosas y mezclas de gramíneas y leguminosas. Documento presentado en Jornada de Fertilización en cultivos y pasturas. INTA. Concepción del Uruguay, Entre Ríos.

Bray, R. H. y Kurtz, L. T. (1945). Determination of total, organic and available forms of phosphorus in soils. Soil Science, 59, 39-45. DOI : http://dx.doi. org/10.1097/00010694-194501000-00006

Ceulemans, T., Merckx, R., Hens, M. y Honnay, O. (2013). Plant species loss from European seminatural grasslands following nutrient enrichment - is it nitrogen or is it phosphorus? Global Ecology and Biogeography, 22, 73-82. DOI: https://doi.org/10.1111/ j.1466-8238.2012.00771.x

Conant, R. T., Paustian, K. y Elliott, E. T. (2001). Grassland management and conversion into grassland: effects on soil carbon. Ecological Applications, 11 (2), 343-355. DOI: https://doi.org/10.1890/10510761(2001)011[0343:GMACIG]2.0.CO;2

del Pino A., Rodríguez, T. y Andion, J. (2015). Production improvement through phosphorus fertilization and legume introduction in grazed native pastures of Uruguay. Journal of Agricultural Science, 154 (2), 347-358. DOI: https://doi.org/10.1017/ S002185961500101X

Dubeux, J. C. B., Sollenberger, L. E., Mathews, B. W., Scholeberg, J. M., y Santos, H. Q. (2007). Nutrient cycling in warm climate grasslands. Crop Science, 47 (3), 915-928. DOI: https://doi.org/10.2135/ cropsci2006.09.0581

García, S., Pezzani, F., Rodríguez, A. y del Pino, A. (2016). Micorrizas en gramíneas nativas: efecto de la fertilización fosfatada a largo plazo. Agrociencia Uruguay, 20, 7-16

Gurevitch, J. y Padilla, D. K. (2004). Are invasive species a major cause of extinctions? Trends in Ecology and Evolution, 19 (9): 470-474. DOI: https://doi. org/10.1016/j.tree.2004.07.005

Gutiérrez Boem, F. H., Alvarez, C., Cabello, M. J., Fernández, P. L., Bono, A., Prystupa, P. y Taboada, M. A. (2008). Phosphorus retention on soil surface of tilled and no-tilled soils. Soil Science Society of America Journal, 72 (4), 1158-1162. DOI: https://doi. org/10.2136/sssaj2007.0189

Harpole, W. S., Sullivan, L. L., Lind, E. M., Firn, J., Adler, P. B., Borer, E. T., ... y Wragg, P. D. (2016). Addition of multiple limiting resources reduces grassland diversity. Nature, 537 (7618), 93-96. DOI: https://doi. org/10.1038/nature19324

Hernández, J., Otegui, O. y Zamalvide, J. P. (1995). Formas y contenidos de fósforo en algunos suelos del Uruguay. (Boletín de Investigaciones $N^{\circ} 43$, Facultad de Agronomía). Montevideo, Uruguay: Universidad de la República.

Isaac, R. A. y Kerber, J. D. (1971). Atomic Absorption and flame photometry: techniques and uses in soil, plant and water analysis. En: L. M. Walsh (Ed.), Instrumental Methods for Analysis of Soils and Plant Tissues (1737). Madison, Winsconsin, Estados Unidos: Soil Science Society of America.

Jaurena, M., Lezama, F., Salvo, L., Cardozo, G., Ayala, W., Terra, J. y Nabinger, C. (2016). The dilemma of improving native grasslands by overseeding legumes: production intensification or diversity conservation. Rangeland Ecology and Management, 69, 35-42. DOI: https://doi.org/10.1016/j.rama.2015.10.006

Kahiluoto, H., Ketoja, E., Vestberg, M. y Saarela, I. (2001). Promotion of AM utilization through reduced $P$ fertilization 2. Field studies. Plant and Soil, 231, 65-79. DOI: https://doi.org/10.1023/A:1010366400009

Karn, J. F. (2001). Phosphorus nutrition of grazing cattle: a review. Animal Feed Science and Technology, 89, (3-4), 133-153. DOI: https://doi.org/10.1016/S03778401(00)00231-5

Koerselman, W. y Meuleman, A. F. (1996). The vegetation $\mathrm{N}: \mathrm{P}$ ratio: a new tool to detect the nature of nutrient limitation. Journal of Applied Ecology, 33 (6): 14411450. DOI:https://doi.org/10.2307/2404783

Koske R. y Gemma J. (1989). A modified procedure for staining roots to detect VA mycorrhizas. Mycological Research, 92 (4), 486-488. DOI: https://doi. org/10.1016/S0953-7562(89)80195-9

Lodge, G. M., King, K. L. y Harden, S. (2006). Effects of pasture treatments on detached pasture litter mass, quality, litter loss, decomposition rates, and residence time in northern New South Wales. Australian Journal of Agricultural Research, 57(10), 1073-1085. DOI: https://doi.org/10.1071/AR05408

Lynch, P. B. (1951). Methods of measuring grassland 
production. Journal of New Zealand Grasslands, 13, 184- 193. DOI: https://doi.org/10.33584/ jnzg.1951.13.957

Mccune, B. y Mefford, M. J. PC-ORD: Multivariate analysis of Ecological Data (Version 4.0 for Windows) [Software]. Gleneden Beach, Oregon, Estados Unidos: MjM Software Design.

Melbourne, B. A., Cornell, H. V., Davies, K. F., Dugaw, C. J., Elmendorf, S., Freestone, ... y Yokomizo $\mathrm{H}$. (2007). Invasion in a heterogeneous world: resistance, coexistence or hostile takeover? Ecology Letters, 10, 77- 94. DOI: https://doi.org/10.1111/j.14610248.2006.00987.x

Millot J. C., Risso, D. y Methol, R. (1987). Relevamiento de pasturas naturales y mejoramientos extensivos en áreas ganaderas del Uruguay. (Informe técnico para la Comisión Honoraria del Plan Agropecuario). Montevideo, Uruguay: Comisión Honoraria del Plan Agropecuario.

Morón, A. (1996). El fósforo en los sistemas productivos: dinámica y disponibilidad en el suelo (I). En A. Morón, D. Martino y J. Sawchik, Manejo y Fertilidad de suelos (Serie Técnica 76, 37-44). Montevideo, Uruguay: Instituto Nacional de Investigación Agropecuaria (INIA).

Mueller - Dombois, D. y Ellenberg, H. (1974). Aims and methods of vegetation ecology. Nueva York, Estados Unidos: Wiley \& Sons.

Murphy, J. y Riley, J. P. (1962). A modified single solution method for determination of phosphate in natural waters. Analytica Chimica Acta. 27, 31-36. DOI: https:// doi.org/10.1016/S0003-2670(00)88444-5

Nelson, D. W. y Sommers, L. E. (1996). Total carbon, organic carbon, and organic matter. En: D. L. Sparks, A. L. Page, P. A. Helmke, R. H. Loeppert, P. N. Soltanpour, M. A. Tabatabai, ...y M. E. Sumner. (Eds.), Methods of Soil Analysis. Part 3. Chemical Methods. (961-1010). Madison, Wisconsin, Estados Unidos: Soil Science Society of America and American Society of Agronomy.

Nelson, D. W. y Sommers, L. E. (1973). Determination of total nitrogen in plant material. Agronomy Journal, 65, 109-112. DOI: https://doi.org/10.2134/agronj1973.000 21962006500010033x

O'Halloran, I. P. (1993). Total and organic phosphorus. En M. R. Carter (Ed.) Soil sampling and methods of analysis. (213-229). Pinawa, Manitoba, Canadá: Canadian Society of Soil Science.

Oehl, F., Laczko, E., Bogenrieder, A., Stahr, K., Bosch, R., Van Der Heijden, M. y Sieverding, E. (2010). Soil type and land use intensity determine the composition of arbuscular mycorrhizal fungal communities. Soil Biology and Biochemistry, 42 (5), 724-738. DOI: https://doi.org/10.1016/j.soilbio.2010.01.006
Pañella, P. G., Cardozo, G., Cuadro, R., Reyno, R. y Lezama, F. (2020). La fertilización fosforada disminuye la riqueza y aumenta el número de especies exóticas de plantas en pastizales intersembrados con leguminosas. Ecología Austral, 30 (3), 331-496.

Paruelo, J. M., Guerschman, J. P., Piñeiro, G., Jobbagy, E. G., Verón, S. R., Baldi, G. y Baeza, S. (2006). Cambios en el uso de la tierra en Argentina y Uruguay: marcos conceptuales para su análisis. Agrociencia, 10 (2), 47-61.

Pascale Medina, C., Heredia, O. y Giufré de López Camelo, L. (2000). Distintas fracciones de fósforo en suelos del norte de Entre Ríos. Revista Facultad de Agronomía, 20, 59-62.

Piñeiro, G., Paruelo, J. M., Jobbágy, E. G., Jackson, R. B. y Oesterheld, M. (2009). Grazing effects on belowground $\mathrm{C}$ and $\mathrm{N}$ stocks along a network of cattle exclosures in temperate and subtropical grasslands of South America. Global Biogeochemical Cycles, 23 (2), GB2003. DOI: https://doi.org/10.1029/2007GB003168

Risso, D. F. (1997). Siembras en el tapiz: consideraciones generales y estado actual de la información en la zona de suelos sobre Cristalino. En M. Carámbula, D. Vaz Martins, E. Indarte (Eds.), Pasturas y Producción Animal en Áreas de Ganadería Extensiva. (Serie Técnica 13, 71-82). Montevideo, Uruguay: Instituto Nacional de Investigación Agropecaria (INIA).

Rubio, G., Cabello, M. J., Gutiérrez Boem, F. H. y Munaro, E. (2008). Estimating available soil phosphorus increases after phosphorus additions in Mollisols. Soil Science Society of America Journal, 72 (6), 17211727. DOI: https://doi.org/10.2136/sssaj2007.0049

Santos-González, J., Finlay, R. y Tehler, A. (2007). Seasonal dynamics of arbuscular mycorrhizal fungal communities in roots in a seminatural grassland. Applied and Environmental Microbiology, 73 (17), 5613-23. DOI: https://doi.org/10.1128/AEM.00262-07

SAS Institute (2009). SAS/STAT 9.2 User's guide. North Carolina, United States: SAS institute.

Sims, J. T. (2009). A phosphorus sorption index. En J. L. Kovar y G. M. Pierzynski (Eds.), Methods for Phosphorus Analysis for Soils, Sediments, Residuals, and Waters (2a ed., Southern Cooperative Series Bulletin No. 408, 20-21). Blacksburg, Virginia, United States: Virginia Tech University

Stewart, C. E., Paustian, K., Conant, R. T., Plante, A. F. y Six, J. (2007). Soil carbon saturation: concept, evidence and evaluation. Biogeochemistry, 86, 19-31. DOI: https://doi.org/10.1007/s10533-007-9140-0

Tilman, D. (1993). Species richness of experimental productivity gradients: how important is colonization limitation? Ecology, 74 (8), 2179-2191. DOI: https:// doi.org/10.2307/1939572 